\title{
A novel linear and broadly neutralizing peptide in the SARS- CoV-2 S2 protein for universal vaccine development
}

\author{
Tuofan $\mathrm{Li}^{1,2,3,4,10}$, Qiuqi Kan ${ }^{1,2,3,4,10}$, Jinying Ge ${ }^{5,6,10}$, Zhimin Wan ${ }^{1,2,3,4,10}$, Mengqi Yuan ${ }^{7}$, Yao Huang ${ }^{8}$, Quan Xie $^{1,2,3,4}$, Yi Yang $^{1,2,3,4}$,

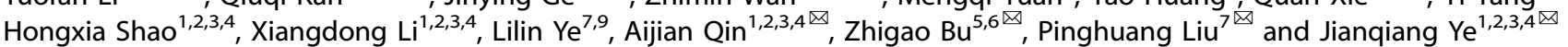

(C) The Author(s) 2021

Cellular \& Molecular Immunology (2021) 18:2563-2565; https://doi.org/10.1038/s41423-021-00778-6

As humans continue to develop COVID-19 widely, numerous novel variants of SARS-CoV-2 have emerged [1, 2]. These variants, which may possess enhanced transmissibility and often result in breakthrough infections in the vaccinated population, pose great challenges to the current vaccine strategies targeting the immunodominance of the receptor-binding domain (RBD) of the spike (S) protein [2].

Similar to SARS-CoV, SARS-CoV-2 uses angiotensin-converting enzyme 2 (ACE2) as the receptor for binding to host cells [3]. During infection, the $S$ protein of SARS-CoV-2 is first cleaved into S1 and S2 subunits; the RBD of the S1 subunit binds to ACE2, and the S2 subunit mediates viral fusion with the cell membrane [3]. In contrast to S1, which is prone to frequent mutation, S2 is highly conserved and can serve as an attractive target for broad protection [4]. However, protective epitopes in the S2 subunit have not yet been extensively characterized $[5,6]$.

In our effort to develop universal SARS-CoV-2 vaccine candidates, four peptides (Table S1) derived from the $S$ protein were synthesized for systematic characterization. In an enzyme-linked immunosorbent assay (ELISA), 11 of 19 serum samples collected from recovered COVID-19 patients recognized the P4 peptide but not the other three (Fig. 1a), indicating that P4 carries potent B cell epitopes.

The $\mathrm{P} 4$ peptide of the S2 subunit, with the amino acid sequence DPLQPELDSFKEELDKYFKNHTSPDVDLGDIS (corresponding to residues $1139-1170$ of the $S$ protein), is located in the linker region between heptad repeat 1 (HR1) and heptad repeat 2 (HR2). The P4 peptide is highly conserved among SARS-CoV, BatCoV RaTG13, SARS-CoV-2 and the recent SARS-CoV-2 variants, including those from lineages B.1.1.7, B.1.1.298, B.1.429, P.2, P.1, B.1.351, and B.1.617.2 (Fig. 1b, c). Because HR1 and HR2 play critical roles in mediating the fusion process of coronavirus [7], we hypothesize that the P4 peptide is a potential target for developing peptidebased inhibitors and vaccines. To identify potential B cell epitopes in P4, we immunized BALB/c mice with the P4 peptide conjugated with $\mathrm{KLH}(\mathrm{P} 4-\mathrm{KLH})$ and generated four monoclonal antibodies (mAbs), designated B10, C10, E2, and G5. These mAbs bound to the S and S2 proteins of both SARS-CoV-2 and SARS-CoV, as revealed by immunofluorescence assays and western blotting (Fig. S1a-c), confirming their specificity for the S2 protein.

To examine neutralization, these mAbs were tested in assays using hACE2/293T cells. mAbs B10, C10, and G5 efficiently neutralized SARS-CoV-2 S-pseudovirus, with half maximal inhibitory concentrations $\left(\mathrm{IC}_{50}\right)$ of $18.05,1.367$, and $2.21 \mu \mathrm{g} / \mathrm{mL}$, respectively (Fig. 1d). Conversely, mAb E2 did not show detectable neutralization of SARS-CoV-2 S-pseudovirus. mAbs B10, C10, and G5 also effectively neutralized SARS-CoV S-pseudovirus, with IC 50 values of $5.295,2.325$, and $2.695 \mu \mathrm{g} / \mathrm{mL}$ (Fig. 1e). Antibody G5 neutralized a SARS-CoV-2 pseudovirus bearing the $\mathrm{S}$ protein from the B.1.351 variant lineage, with an $\mathrm{IC}_{50}$ of $2.608 \mu \mathrm{g} / \mathrm{mL}$ (Fig. 1f). In line with these data, mAbs B10, C10, and G5 (tested with mouse ascites containing mAbs) were able to neutralize wild-type SARSCoV-2 (Fig. 1g). Moreover, mAbs B10, C10, and G5, but not mAb E2 and the control $\mathrm{mAb}$, inhibited the spread of a vesicular stomatitis virus expressing SARS-CoV-2 $\mathrm{S}$ and green fluorescent proteins (VSV-SARS-CoV2-S-GFP) in Vero E6 cells (Fig. 1h). Moreover, immunoprecipitation assays revealed that mAbs B10, C10, and G5 efficiently immunoprecipitated both the S and S2 proteins of SARS-CoV-2 but that mAb E2 only immunoprecipitated the $S$ protein of SARS-CoV-2 at low efficiency (Fig. 1i).

Western blotting using truncated $\mathrm{P} 4$ demonstrated that all the mAbs reacted with only S2-P4 and S-P4-1 and not the other truncations (Table S2 and Fig. S1d,e), which indicates that the epitopes recognized by these mAbs are located in ${ }_{1147}$ SFKEELDKYFKNHTSP $_{1162}$ in S2 and that the eight amino acids in ${ }_{1155}$ YFKNHTSP $_{1162}$ are critical for the epitopes. Whether these mAbs recognize the same epitope or different epitopes needs to be further elucidated. To evaluate whether the epitope in the P4 peptide is of value for vaccine development, a trimeric P4 expression vector, $3 \times \mathrm{P} 4$, was constructed for protein expression (Table S3 and Fig. S2a, b). Trimeric P4 was readily recognized by $\mathrm{mAb}$ G5, highlighting the antigenicity of the expressed trimeric P4 protein (Fig. 1j). In addition, sera from mice immunized with the purified trimeric P4 protein neutralized SARS-CoV-2 S-pseudovirus

\footnotetext{
'Jiangsu Coinnovation Center for Prevention and Control of Important Animal Infectious Diseases and Zoonoses, Yangzhou University, Yangzhou 225009 Jiangsu, China. ${ }^{2}$ Key Laboratory of Jiangsu Preventive Veterinary Medicine, Key Laboratory for Avian Preventive Medicine, Ministry of Education, College of Veterinary Medicine, Yangzhou University, Yangzhou 225009 Jiangsu, China. ${ }^{3}$ Joint International Research Laboratory of Agriculture and Agri-Product Safety, the Ministry of Education of China, Yangzhou University, Yangzhou 225009 Jiangsu, China. ${ }^{4}$ Institutes of Agricultural Science and Technology Development, Yangzhou University, Yangzhou 225009 Jiangsu, China. ${ }^{5}$ State Key Laboratory of Veterinary Biotechnology, Harbin Veterinary Research Institute, Chinese Academy of Agricultural Sciences, 150069 Harbin, China. ${ }^{6}$ National High Containment Laboratory for Animal Diseases Control and Prevention, 150069 Harbin, China. ${ }^{7}$ College of Veterinary Medicine, China Agricultural University, 100193 Beijing, China. ${ }^{8}$ Center of Diseases Control and Prevention, Yangzhou 225009 Jiangsu, China. ${ }^{9}$ Institute of Immunology, PLA, Third Military Medical University, 400038 Chongqing, China. ${ }^{10}$ These authors contributed

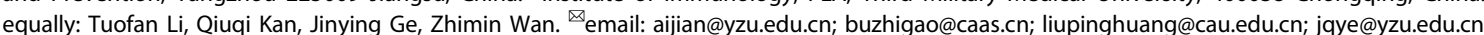

Received: 11 September 2021 Accepted: 18 September 2021

Published online: 13 October 2021 
a

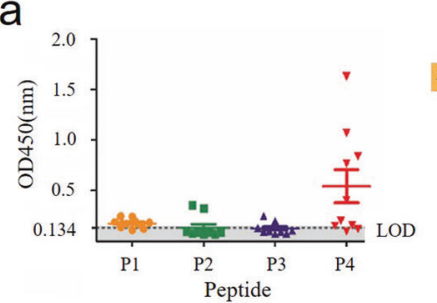

d

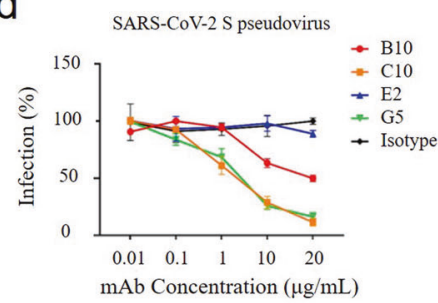

f

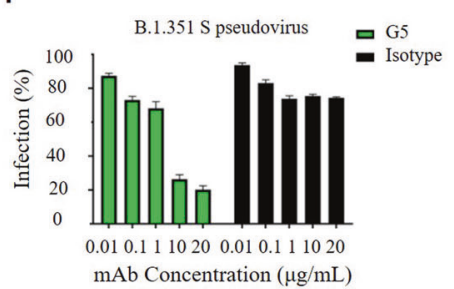

b

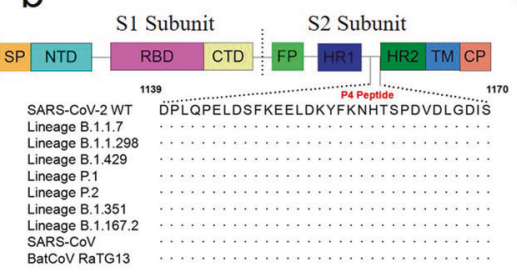

e

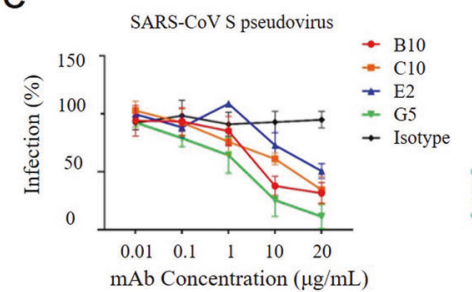

C
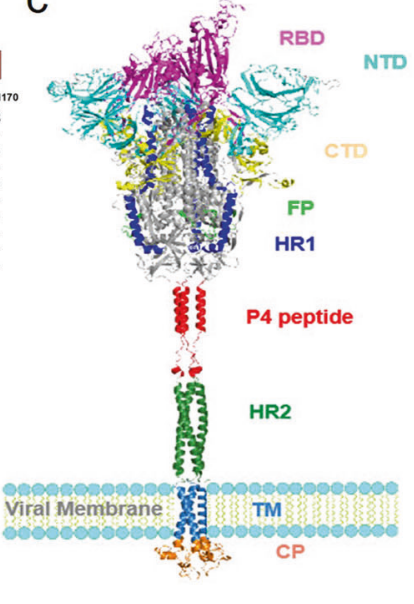

g
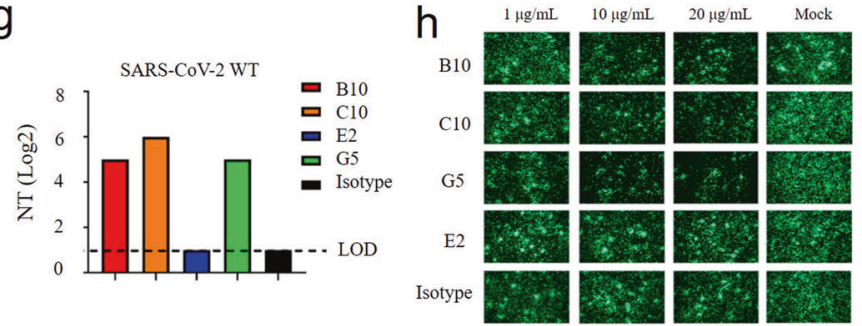

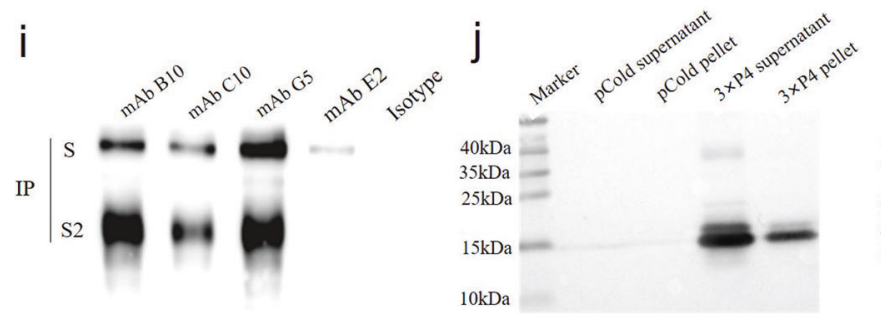

k
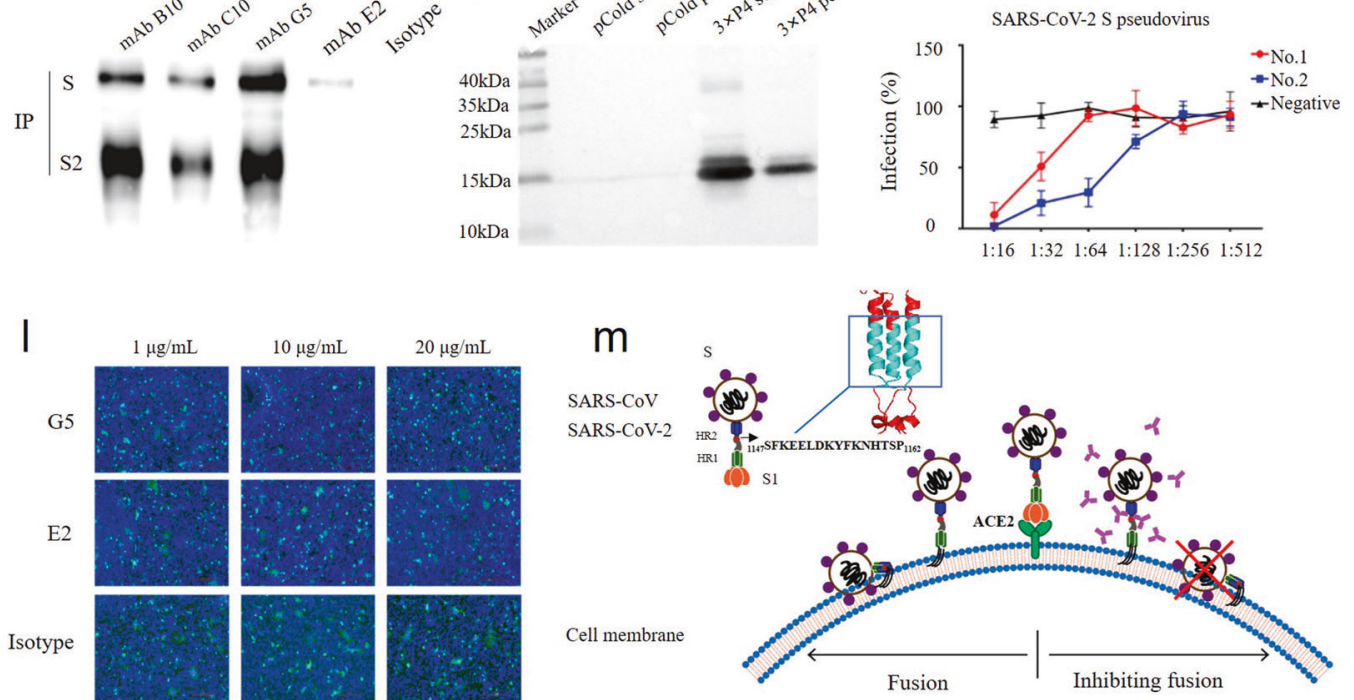

Fig. 1 A novel linear and broadly neutralizing peptide in the S2 protein of SARS-CoV-2. a ELISA with the indicated peptides as antigens was used to test sera from recovered COVID-19 patients. LOD, limit of detection. $\mathbf{b}$ Sequence alignment showing the location of P4 in SARSCoV-2, SARS-CoV, BatCoV RaTG13 and SARS-CoV-2 variants. c A 3D structure model diagram of the SARS-CoV-2 S protein generated with PyMOL based on PDB 6VSB_1_1_1 [10]. The P4 peptide is shown in red. $\mathbf{d}$ and e Neutralizing activity of B10, C10, E2, and G5 mAbs against SARS-CoV-2 S pseudovirus (d) and SARS-CoV S pseudovirus (e). f Neutralizing activity of mAb G5 against the SARS-CoV-2 S pseudovirus B.1.351 South Africa variant. In (d-f), measurements were performed using purified mAbs and SARS-CoV-2 and SARS-CoV S pseudoviruses in hACE2/293T cells, and the results are shown as the percentage of inhibition (mean \pm SD). $\mathbf{g}$ Neutralizing activity of B10, C10, E2, and G5 mAbs against wild-type SARS-CoV-2. The assay was performed with mouse ascites and wild-type SARS-CoV-2 in Vero E6 cells, and the data are expressed as the log2 number of dilution. $\mathbf{h}$ Inhibition of S protein-mediated virus spread by mAbs. i Affinities of B10, C10, E2, and G5 mAbs for the S protein were analyzed by immunoprecipitation assays. 293T cells were transfected with a plasmid carrying the SARS-CoV-2 S gene, and $\mathrm{mAbs} B 10, \mathrm{C10}, \mathrm{E2}$, and G5 were used as the capture antibody to perform immunoprecipitation, followed by western blot analysis using $\mathrm{mAb}$ G5. $\mathbf{j}$ Identification of the trimeric P4 protein by western blotting. The supernatants and pellets from lysates of BL21 cells transformed with $3 \times \mathrm{P} 4$ or the pColdl vector were analyzed by western blotting using mAb G5 as the primary antibody. k Neutralization of SARS-CoV-2 pseudovirus by sera from mice immunized with the trimeric P4 protein. The assay was performed with mouse sera and SARS-CoV-2 pseudovirus in hACE2/293T cells. I Inhibition of S-mediated cell-cell fusion by mAbs. $\mathbf{m}$ A schematic diagram showing the potential mechanism by which mAbs B10, C10, E2, and G5 neutralize SARS-CoV-2/SARS-CoV. mAbs against ${ }_{1147}$ SFKEELDKYFKNHTSP $_{1162}$ inhibit SARSCoV-2/SARS-CoV viral entry by inhibiting fusion between the virus and cell membrane 
in a dose-dependent manner (Fig. 1k). These data demonstrate that trimeric P4 is a potential vaccine candidate for broad protection against SARS-CoV-2.

To further investigate the mechanism underlying inhibition of SARS-CoV-2 by these mAbs, we performed a cell-cell fusion inhibition assay, as previously described [7]. $\mathrm{mAb} \mathrm{G} 5$, but not E2 or the control mAb, inhibited SARS-CoV-2-S-mediated cell-cell fusion in a dose-dependent manner (Fig. 1I). Thus, antibodies against P4 might inhibit viral entry by blocking fusion of the virus with the host cell membrane.

In conclusion, we identified a novel linear and broadly neutralizing peptide in the S2 protein of SARS-CoV-2. Different from other crossneutralizing epitopes [4-9], this linear peptide ( ${ }_{1147}$ SFKEELDKYFKNHTSP $_{1162}$ ) is conserved across SARS-CoV, BatCoV RaTG13, SARS-CoV-2, and SARS-CoV-2 variants. mAbs targeting this peptide efficiently neutralized SARS-CoV-2 and SARS-CoV S-pseudovirus as well as wild-type SARS-CoV-2. These antibodies also inhibited both S-mediated cell-cell membrane fusion and viral spread among cells. Furthermore, the recombinant trimeric P4 carrying the ${ }_{1147}$ SFKEELDKYFKNHTSP $_{1162}$ epitope induced neutralizing antibodies against SARS-CoV-2 S-pseudovirus. Taken together, these findings suggest that antibodies targeting the peptide ${ }_{1147}$ SFKEELDKYFKNHTSP ${ }_{1162}$ may neutralize both SARS-CoV-2 and SARS-CoV by preventing fusion between the virus and cell membrane (Fig. $1 \mathrm{~m}$ ). In light of the rapid emergence of antigenic variants of SARS-CoV-2, the identification of neutralizing peptides such as ${ }_{1147}$ SFKEELDKYFKNHTSP ${ }_{1162}$ represents an opportunity for the development of universal vaccines and therapeutic reagents against current pandemic strains as well as future SARS-CoV-2 mutants.

\section{DATA AVAILABILITY}

The datasets used and/or analyzed during the current study are available from the corresponding author on reasonable request.

\section{REFERENCES}

1. Zhou $P$, Yang $X L$, Wang $X G$, Hu B, Zhang L, Zhang W, et al. A pneumonia outbreak associated with a new coronavirus of probable bat origin. Nature. 2020;579:270-3.

2. Greaney AJ, Starr TN, Gilchuk P, Zost SJ, Binshtein E, Loes AN, et al. Complete Mapping of Mutations to the SARS-CoV-2 Spike Receptor-Binding Domain that Escape Antibody Recognition. Cell Host Microbe. 2021;29:44-57.

3. Hoffmann M, Kleine-Weber H, Schroeder S, Krüger N, Herrler T, Erichsen S, et al. SARS-CoV-2 cell entry depends on ACE2 and TMPRSS2 and is blocked by a clinically proven protease inhibitor. Cell. 2020;181:271-80.

4. Sauer MM, Tortorici MA, Park YJ, Walls AC, Homad L, Acton OJ, et al. Structural basis for broad coronavirus neutralization. Nat Struct Mol Biol. 2021;28:478-86.

5. Li Y, Lai DY, Zhang HN, Jiang HW, Tian X, Ma ML, et al. Linear epitopes of SARSCoV-2 spike protein elicit neutralizing antibodies in COVID-19 patients. Cell Mol Immunol. 2020;17:1095-7.

6. Li Y, Ma M, Lei Q, Wang F, Hong W, Lai D, et al. Linear epitope landscape of the SARS-CoV-2 Spike protein constructed from 1,051 COVID-19 patients. Cell Rep. 2021;34:108915

7. Xia S, Liu M, Wang C, Xu W, Lan Q, Feng S, et al. Inhibition of SARS-CoV-2 (previously 2019-nCoV) infection by a highly potent pan-coronavirus fusion inhibitor targeting its spike protein that harbors a high capacity to mediate membrane fusion. Cell Res. 2020;30:343-55.

8. Ladner JT, Henson SN, Boyle AS, Engelbrektson AL, Fink ZW, Rahee F, et al. Epitope-resolved profiling of the SARS-CoV-2 antibody response identifies crossreactivity with endemic human coronaviruses. Cell Rep Med. 2021;2:100189.
9. Song G, He WT, Callaghan S, Anzanello F, Huang D, Ricketts J, et al. Cross-reactive serum and memory B-cell responses to spike protein in SARS-CoV-2 and endemic coronavirus infection. Nat Commun. 2021;12:2938.

10. Woo H, Park SJ, Choi YK, Park T, Tanveer M, Cao Y, et al. Developing a Fully Glycosylated Full-Length SARS-CoV-2 Spike Protein Model in a Viral Membrane. J Phys Chem B. 2020;124:7128-37.

\section{ACKNOWLEDGEMENTS}

This study was supported by the Key Laboratory of Prevention and Control of Biological Hazard Factors (Animal Origin) for Agrifood Safety and Quality (26116120), Social Development Project of the Yangzhou Science and Technology (YZ2020122), the Research Foundation for Talented Scholars in Yangzhou University and the Priority Academic Program Development of Jiangsu Higher Education Institutions.

\section{AUTHOR CONTRIBUTIONS}

$J Y, A Q, P L$, and $Z B$ designed the project. $T L, Q K, J G, Z W, M Y, Y H, Q X, P L, H S$, and $Y Y$ carried out the experiments. TL, QK, JG, $\mathrm{XL}, \mathrm{LY}, \mathrm{ZB}$, and $\mathrm{AQ}$ analyzed the data. $\mathrm{TL}, \mathrm{QK}$ and JY drafted the paper. JY supervised all the experiments and participated in the data analysis. $\mathrm{TL}, \mathrm{JY}, \mathrm{PL}, \mathrm{AQ}$ and $\mathrm{XL}$ discussed and prepared the final report. All of the authors have read and approved the final paper.

\section{COMPETING INTERESTS}

The authors declare no competing interests.

\section{ETHICS APPROVAL AND CONSENT TO PARTICIPATE}

All animal experiments were performed in accordance with the institutional animal care guidelines approved by the Animal Care Committee of Yangzhou University, China.

\section{ADDITIONAL INFORMATION}

Supplementary information The online version contains supplementary material available at https://doi.org/10.1038/s41423-021-00778-6.

Correspondence and requests for materials should be addressed to Aijian Qin, Zhigao Bu, Pinghuang Liu or Jianqiang Ye.

Reprints and permission information is available at http://www.nature.com/ reprints

\footnotetext{
Open Access This article is licensed under a Creative Commons

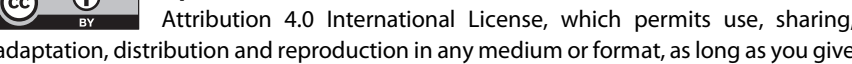
adaptation, distribution and reproduction in any medium or format, as long as you give
appropriate credit to the original author(s) and the source, provide a link to the Creative Commons license, and indicate if changes were made. The images or other third party material in this article are included in the article's Creative Commons license, unless indicated otherwise in a credit line to the material. If material is not included in the article's Creative Commons license and your intended use is not permitted by statutory regulation or exceeds the permitted use, you will need to obtain permission directly from the copyright holder. To view a copy of this license, visit http://creativecommons. org/licenses/by/4.0/.
}

(c) The Author(s) 2021 